Supporting Information

\title{
Chiral Chemistry of Homochiral Porous Thin Film with Different Growth Orientations
}

Shu-Mei Chen ${ }^{\dagger}$,Min Liu ${ }^{\dagger+}$, Zhi-Gang Gu*t, Wen-Qiang Fu ${ }^{\dagger}$, and Jian Zhang ${ }^{*}$

${ }^{\dagger}$ College of Chemistry, Fuzhou University, Fuzhou, Fujian 350108, PR China

State Key Laboratory of Structural Chemistry, Fujian Institute of Research on the Structure of Matter, Chinese Academy of Sciences, Fuzhou, Fujian 350002, P.R. China. 


\section{Preparation of MUD and MHDA SAMs}

Briefly, an Au substrate with a thickness of $150 \mathrm{~nm}$ was deposited onto a $\mathrm{Si}(100)$ substrate with a thickness of $2 \mathrm{~nm}$ pre-coated Ti layer, or commercially available quartz crystal microbalance (QCM) sensor coated with $\mathrm{Au}$ was immersed into a $1 \mathrm{mM}$ ethanolic solution of 11-mercapto-1-undecanol (MUD) for 24 hours or a $20 \mu \mathrm{M}$ ethanolic solution of 16-mercaptohexadecanoic acid (MHDA) with acetic acid (10\% in volume) for 48 hours. All the samples are rinsed with pure ethanol and dried under nitrogen flux before the preparation of sample.

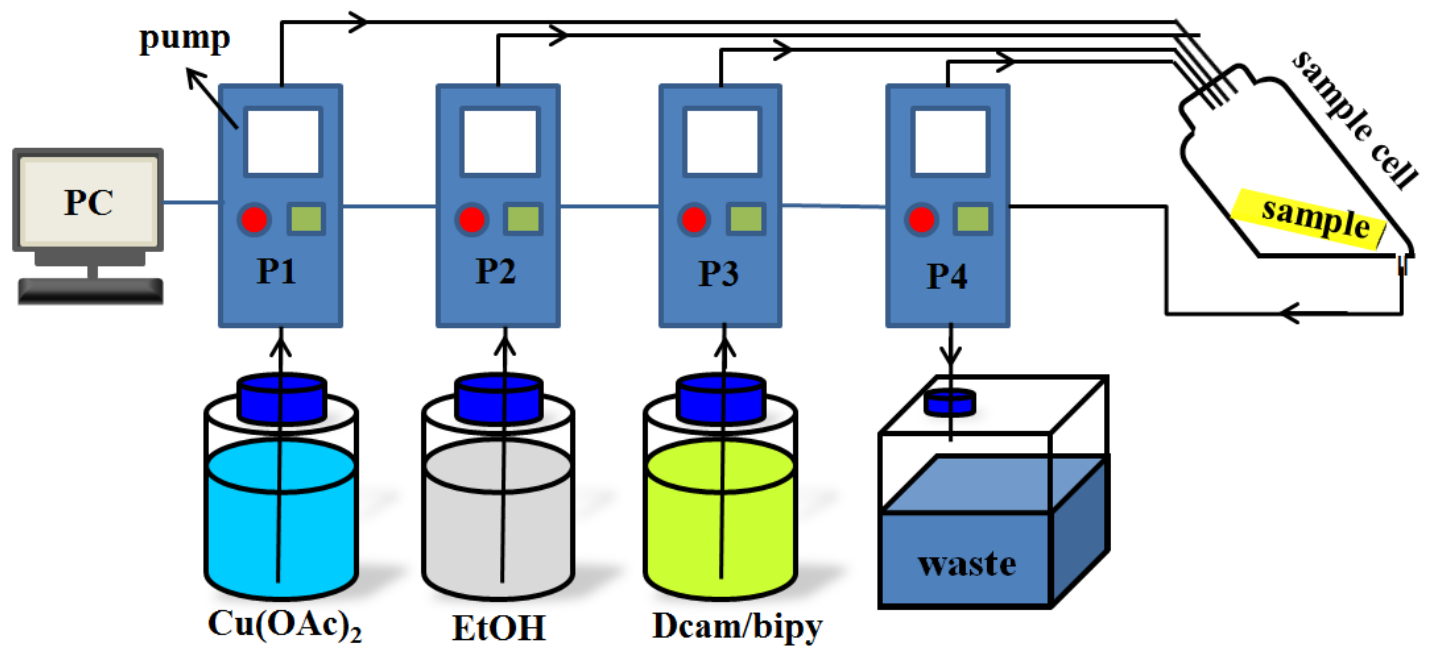

Figure S1. The LPE pump setup of SURchirMOF-1 prepared by $\mathrm{Cu}(\mathrm{OAC})_{2}$ and Dcam/bipy solutions. 

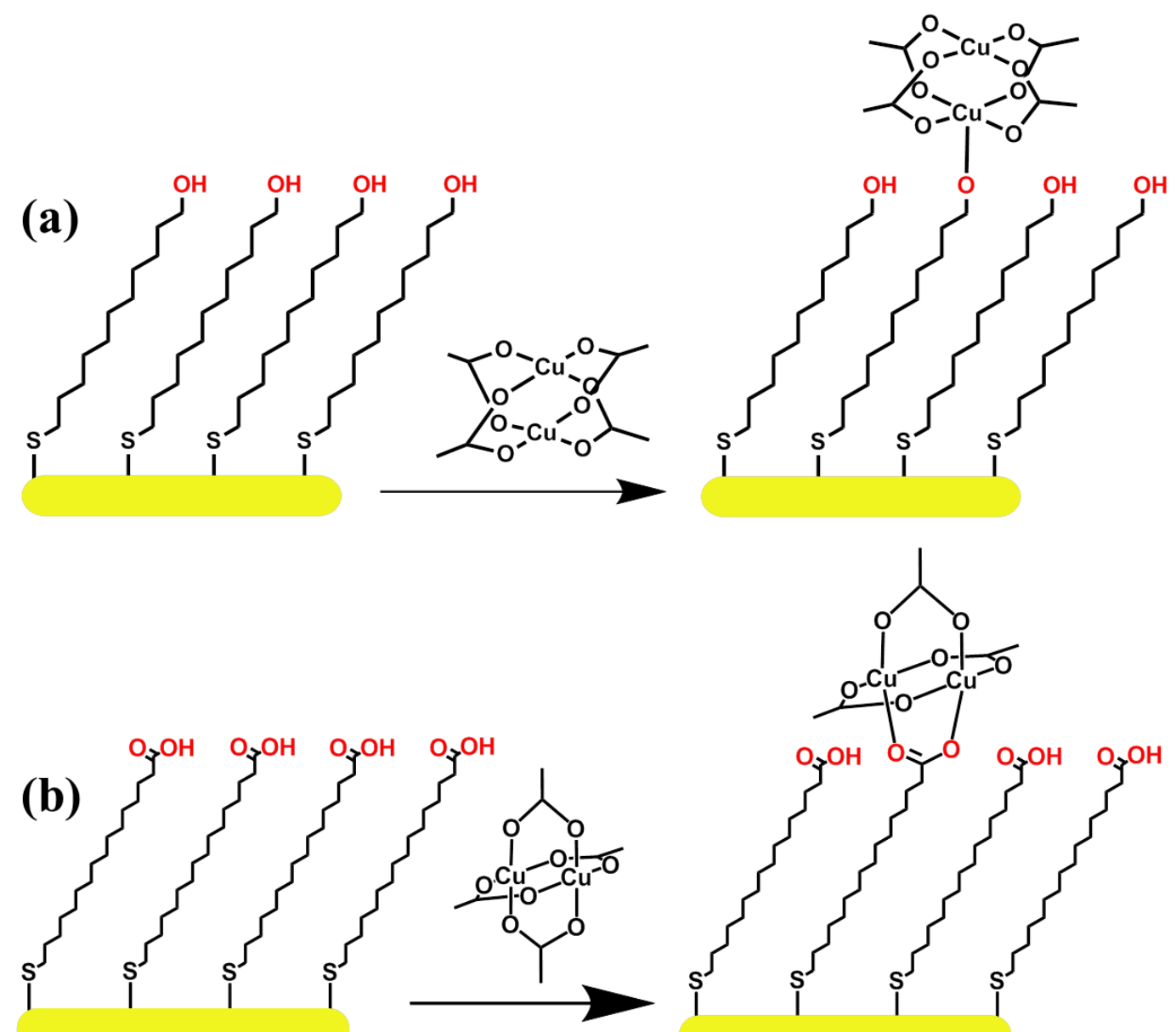

Figure S2. Growth mechanism of $\mathrm{Cu}(\mathrm{OAc})_{2}$ in the first step of $\left[\mathrm{Cu}_{2} \mathrm{Dcam}_{2} \text { bipy }\right]_{\mathrm{n}}$ on (a) MUD and (b) MHDA SAMs. 

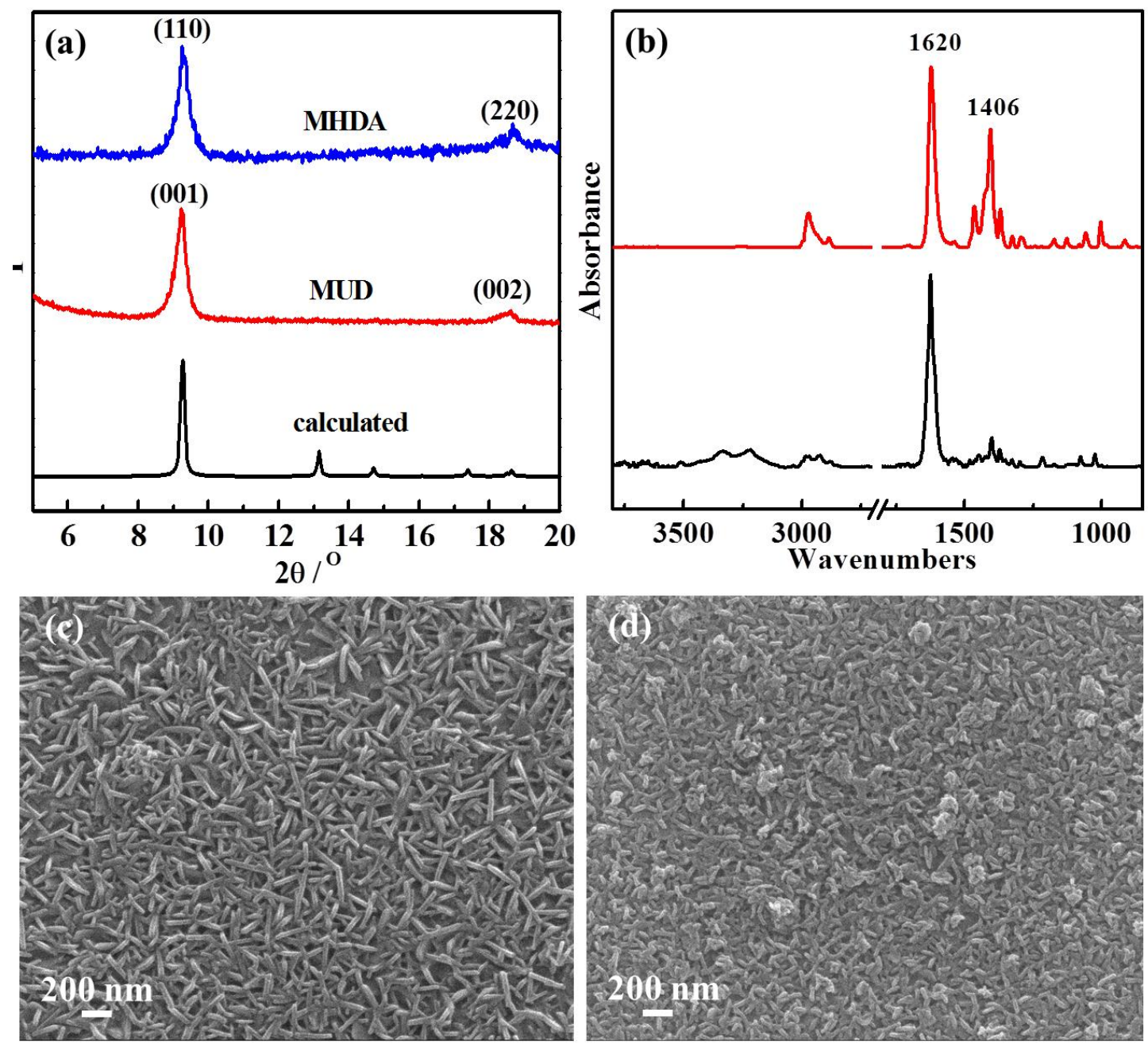

Figure S3. the out plane XRD (a) and IRRAS (b) of SURchirMOF-2 grown on MUD and MHDA; the SEM images of SURchirMOF-2 grown on MUD (c) and $\operatorname{MHDA}(\mathrm{d})$. 
(a)

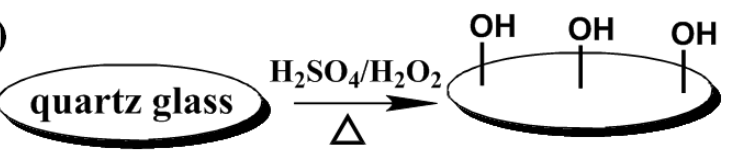

functionalized $\mathrm{OH}[001]$ on quartz glass

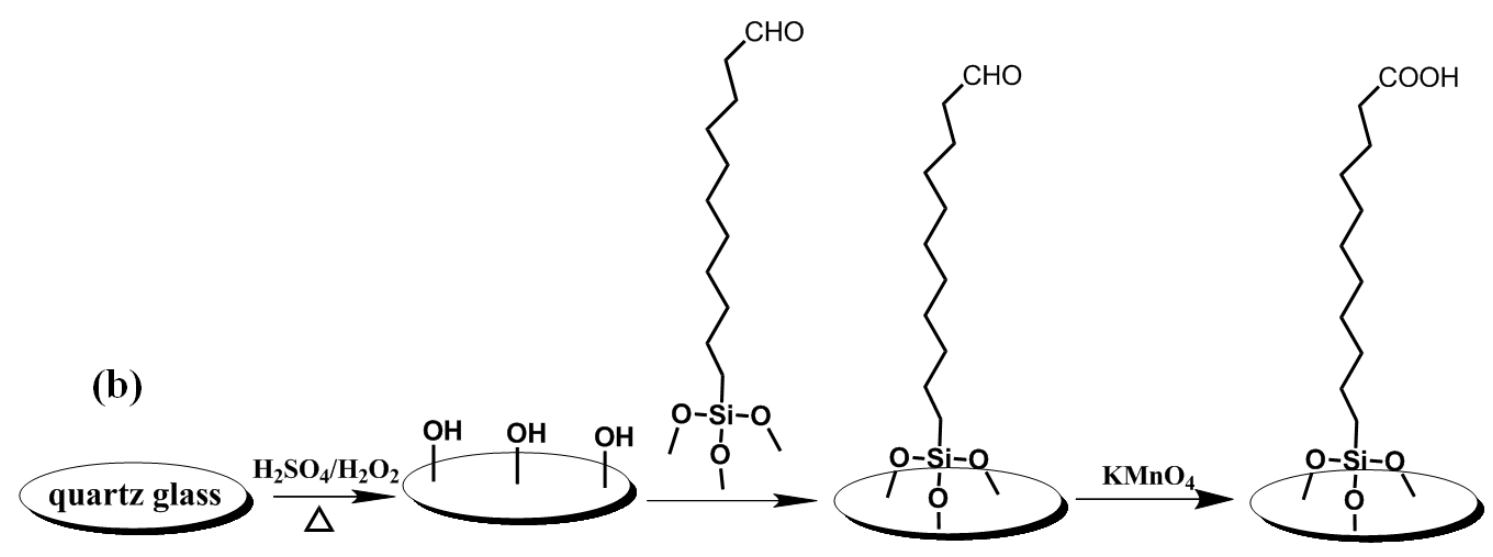

functionalized $\mathrm{COOH}[110]$ on quartz glass

Figure S4. The preparation of hydroxyl- (a) and carboxyl-functionalized (b) quartz glasses.

(a)

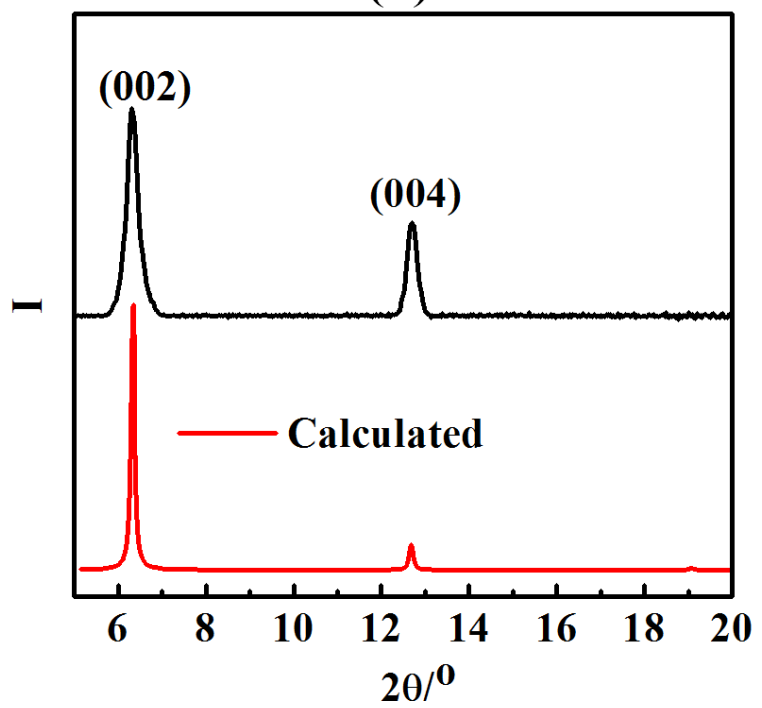

(b)

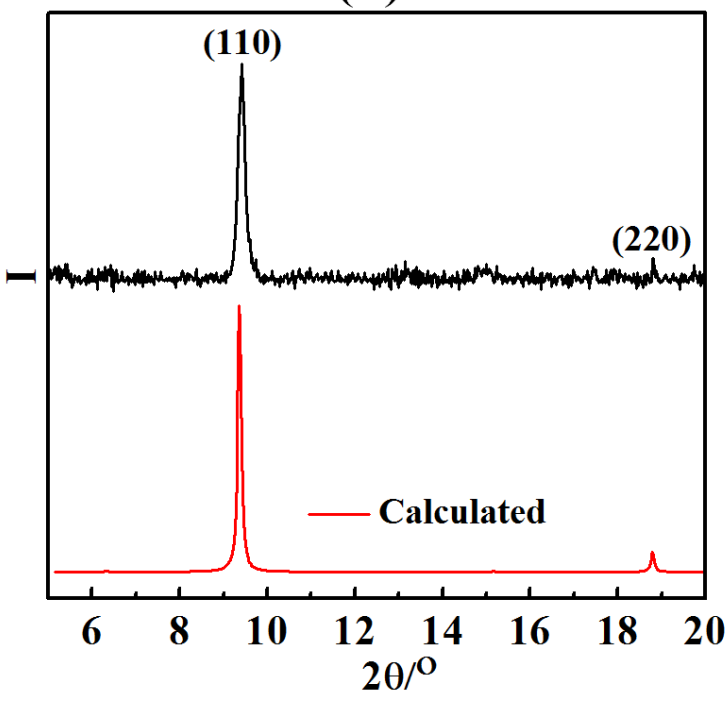

Figure S5. The out plane XRD of SURchirMOF-1 gronw on hydroxyl- (a) and carboxyl-functionalized (b) quartz glasses. 


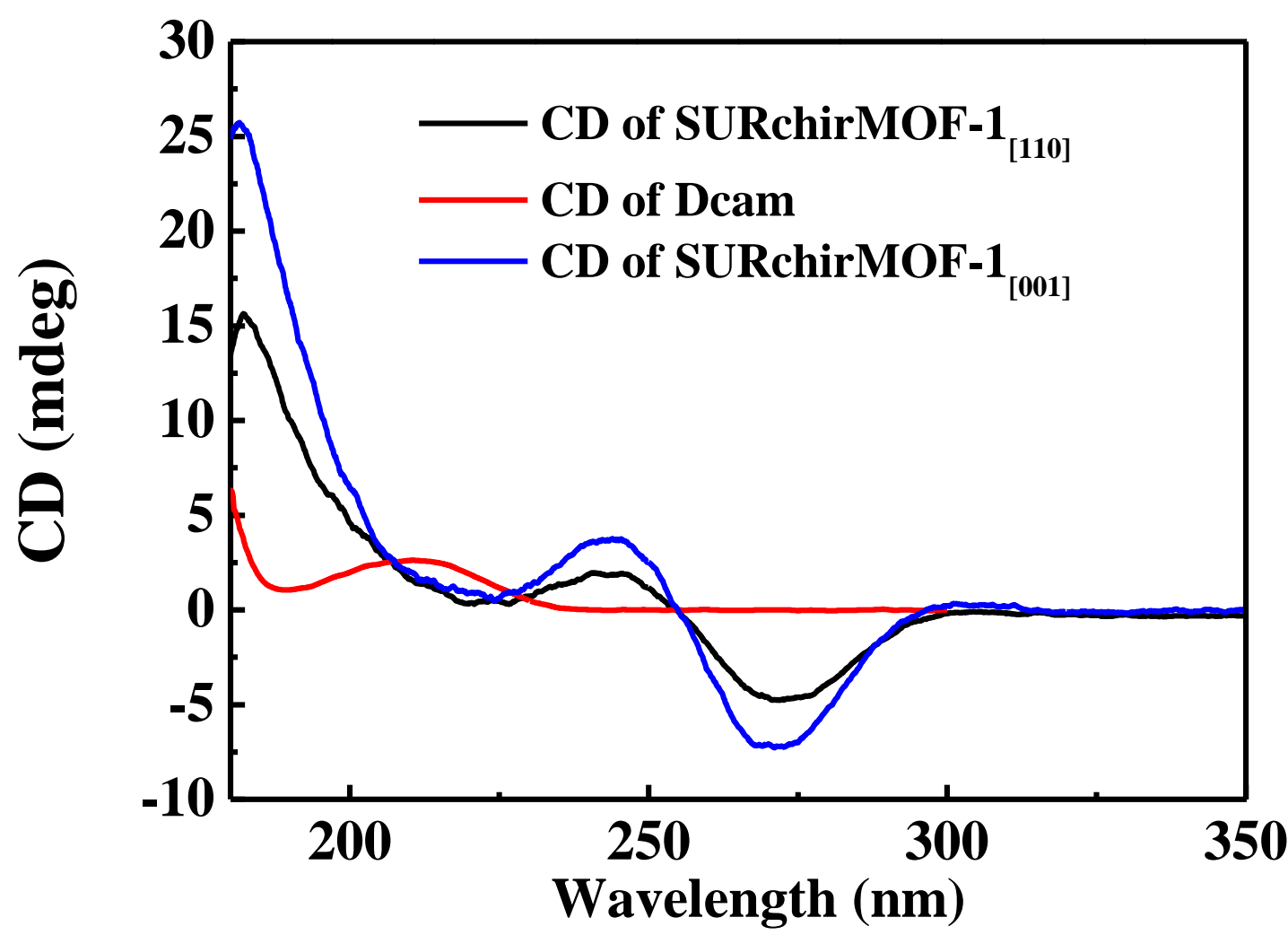

Figure S6. The CD spectra of Dcam ligand and SURchirMOF-1 [001] and SURchirMOF-1 $\mathbf{1}_{[110]}$. 


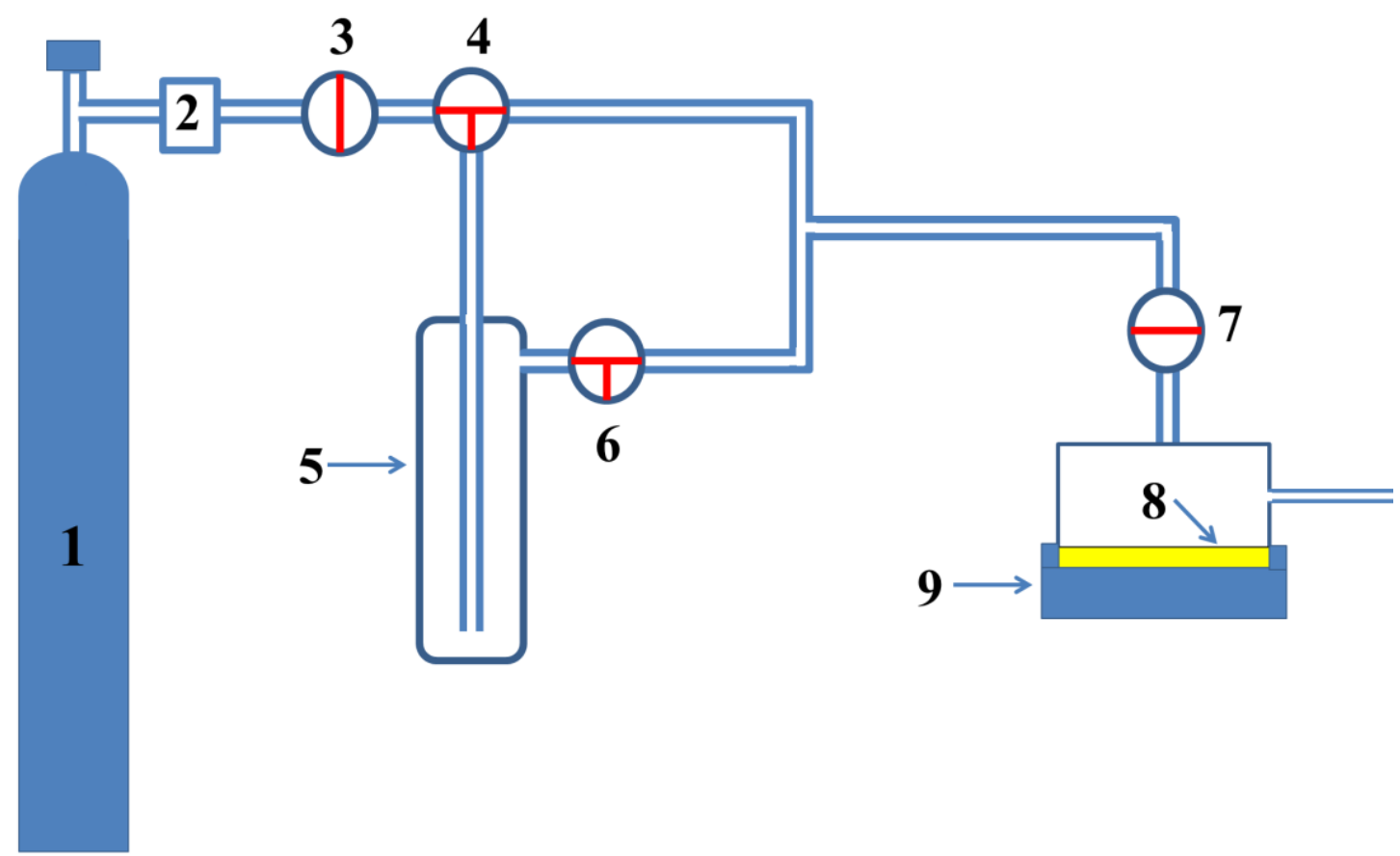

Figure S7. Gas phase QCM setup employed for the sorption measurements: (1) gas (N2) supply, (2) gas flow controller, (3) main valve, (4) three-way valve, (5) storage container with the loading substance, $(6,7)$ outlet valves, (8) QCM sensor, (9) sample holder with electric feed through. 

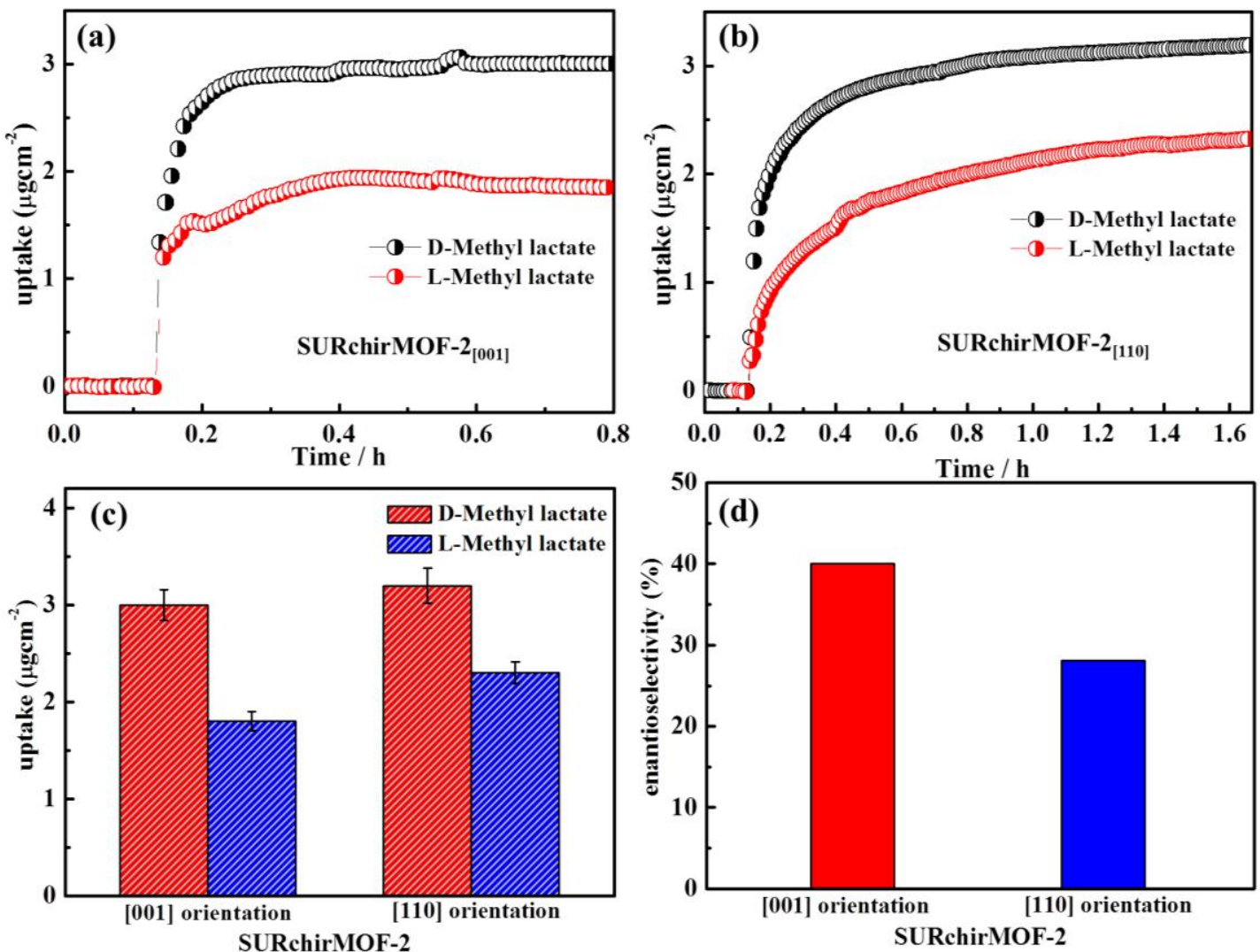

Figure S8. the D- and L-methyl lactate uptake of SURchirMOF-2 grown on MUD (a) and MHDA SAMs substrates (b); the bar graph of reproduced mass uptake (c) and enantioselectivity (d) of SURchirMOF-2 grown on MUD and MHDA SAMs substrates. 

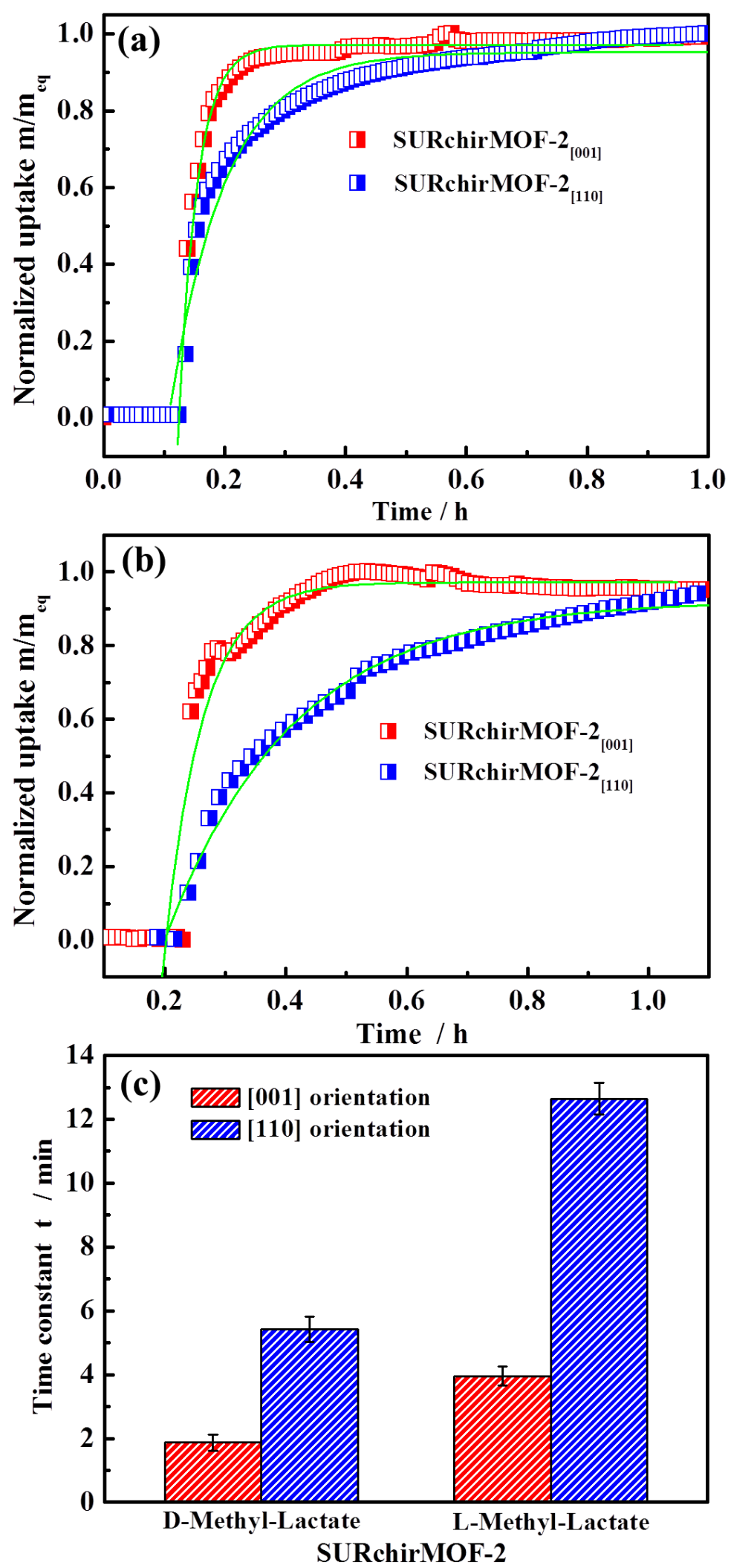

Figure S9. the normalized uptake of D- (a) and L-methyl lactate (b) uptake of SURchirMOF-2 grown along [001] and [110]; (c) the bar graph of time constant of D- and L-methyl lactate in SURchirMOF-2 with [001] and [110] orientations. 